\title{
Detection of long-chain non-encoding RNA differential expression in non-small cell lung cancer by microarray analysis and preliminary verification
}

\author{
YUMIN WANG ${ }^{1,2^{*}}$, GANG XU $^{1 *}$, WEI CHEN ${ }^{2}$, QINSHI PAN ${ }^{1}$, KATE HUANG $^{3}$, \\ JINGYE PAN ${ }^{4}$, WENHUI ZHANG ${ }^{1}$ and JIE CHEN ${ }^{4}$
}

\begin{abstract}
${ }^{1}$ Department of Laboratory Medicine, The First Affiliated Hospital of Wenzhou Medical University, Wenzhou, Zhejiang 325000; ${ }^{2}$ Center for Laboratory Medicine, The First Affiliated Hospital, School of Medicine, Xi'an Jiaotong University, Xi'an, Shaanxi 710061; ${ }^{3}$ Department of Pathology and ${ }^{4}$ Intensive Care Unit, The First Affiliated Hospital of Wenzhou Medical University, Wenzhou, Zhejiang 325000, P.R. China
\end{abstract}

Received March 20, 2014; Accepted October 24, 2014

DOI: $10.3892 / \mathrm{mmr} .2014 .2944$

\begin{abstract}
Long-chain non-coding RNAs (lncRNAs) have been shown to be involved in the development and progression of non-small cell lung cancer (NSCLC). However, the roles of lncRNAs in NSCLC are not well understood. In this study, a high-throughput microarray was used to compare the IncRNA and mRNA expression profiles in NSCLC and normal tissue (NT) samples. Several candidate adenocarcinoma-associated lncRNAs were verified by reverse transcription-quantitative polymerase chain reaction (RT-qPCR). Using abundant and varied probes, we were able to assess 30,586 lncRNAs and 26,109 coding transcripts in our microarray. It was observed that 1,242 lncRNAs and 1,102 mRNAs were differentially expressed ( $\geq 2$-fold change) in NSCLC compared with NT samples, indicating that numerous IncRNAs were significantly upregulated or downregulated in NSCLC. We also observed via RT-qPCR that 10 lncRNAs were aberrantly expressed in NSCLC compared with histologically matched normal lung tissues. Among these, RP11-385J1.2 and TUBA4B were the most aberrantly expressed lncRNAs, as estimated by RT-qPCR in 90 pairs of NSCLC and NT samples. In conclusion, the present study detected the IncRNA expression patterns in
\end{abstract}

Correspondence to: Mr. Qinshi Pan, Department of Laboratory Medicine, The First Affiliated Hospital of Wenzhou Medical University, Fuxue Xian 2, Wenzhou, Zhejiang 325000, P.R. China E-mail: pqs122@163.com

Mr. Jie Chen, Intensive Care Unit, The First Affiliated Hospital of Wenzhou Medical University, Fuxue Xian 2, Wenzhou, Zhejiang 325000, P.R. China

E-mail: chenjie991300@163.com

*Contributed equally

Key words: non-small-cell lung cancer, long-chain non-encoding RNAs, microarray
NSCLC by microarray. The results revealed that a number of lncRNAs were differentially expressed in NSCLC tissues, suggesting that they may play a key role in tumor development.

\section{Introduction}

The global lung cancer mortality rate is the highest among all types of cancer and its incidence is gradually increasing (1). Non-small cell lung cancer (NSCLC) is the most common type of lung cancer (accounting for $80 \%$ of all cases), and includes squamous cell carcinoma, adenocarcinoma and large cell carcinoma. Although surgical resection, radiation therapy and chemotherapy technology continue to improve gradually, patients with lung cancer remain exceedingly vulnerable to relapse and mortality (2). The global cure rate of lung cancer is low and the average 5-year survival rate is $<15 \%$ (3-6). However, the mechanisms of NSCLC have not been elucidated, and hence the study of NSCLC is crucial.

Long-chain non-coding RNAs (long non-coding RNAs, lncRNAs) are RNA molecules with a transcript longer than 200 nucleotides in the nucleus or cytoplasm (7). LncRNAs are usually divided into five categories: Sense, antisense, bidirectional, introns and intergenic lncRNAs. In recent years, a large number of lncRNAs have been identified and a human lncRNA database providing details of lncRNA expression and other significant information has been established (8). Numerous studies have linked the lncRNAs with diseases, and abnormal expression has been noted in a range of diseases, including cancer $(9,10)$.

Studies have demonstrated that lncRNAs are differentially expressed in normal cells and tumor cells, and since lncRNAs are a significant regulatory factor of gene expression, their aberrant expression will inevitably lead to abnormalities in gene expression and tumorigenesis. LncRNA disorders are also a feature of several types of cancer and promote the development, invasion and metastasis of tumors by a variety of mechanisms $(9,11)$. LncRNAs regulate the transcriptional expression at the epigenetic, transcription and post-transcription levels (12-14). 
Previous studies have demonstrated that lncRNAs are involved in the development and progression of NSCLC. However, research into IncRNAs in NSCLC is in its infancy and only a small number of NSCLC-associated lncRNAs have been identified, including lcRNA HOTAIR, lcRNA H19, lcRNA ANRIL, lcRNA MALAT1 $(15,16)$ and lcRNA SCAL1 (17), lncRNA AK126698 (18) and lncRNA GAS6-AS1 (19). However, IncRNAs of NSCLC require further study to elucidate their mechanism of action.

In this study, we detected the lncRNA and mRNA expression patterns in NSCLC samples compared with corresponding adjacent normal tissue (NT) samples, several of which were evaluated by reverse transcription-quantitative polymerase chain reaction (RT-qPCR) in a total of 90 pairs of tissues. The results revealed that 1 cRNA expression patterns may provide new molecular biomarkers for the diagnosis of NSCLC.

\section{Materials and methods}

Patient samples. NSCLC and corresponding NT samples were prospectively collected from 105 patients at The First Affiliated Hospital of Wenzhou Medical University, China, from April 2012 to August 2013. Samples from 15 of the patients were used for microarray analysis of 1ncRNAs and those from the remaining 90 were used for additional evaluations (Table I). The diagnosis of adenocarcinoma was confirmed by the histopathological results. The NSCLC and matched NT samples were snap-frozen in liquid nitrogen immediately after resection. The study was approved by the Institutional Ethics Review Board of The First Affiliated Hospital of Wenzhou Medical University, and all patients provided written informed consent for this study.

RNA extraction. NSCLC cells were obtained by laser microdissection; the proportion of cancer cells in the tissue sections was $100 \%$. The 15 NSCLC specimens were divided into three groups; namely, every five samples from NSCLC were combined into a group. Next, 15 of the corresponding NT samples were mixed into one group. The four groups were subjected to RNA extraction. Total RNA was extracted using TRIzol reagent (Invitrogen Life Technologies, Carlsbad, CA, USA), according to the manufacturer's instructions. The integrity of the RNA was assessed by electrophoresis on a denaturing agarose gel. An ND-1000 spectrophotometer (NanoDrop Technologies, Inc., Wilmington, DE, USA) was used for the accurate measurement of RNA concentration $\left(\mathrm{OD}_{260}\right)$, protein contamination $\left(\mathrm{OD}_{260} / \mathrm{OD}_{280}\right.$ ratio) and organic compound contamination $\left(\mathrm{OD}_{260} / \mathrm{OD}_{230}\right.$ ratio).

Microarray and computational analysis. An Agilent array platform (Agilent Technologies, Inc., Santa Clara, CA, USA) was employed for microarray analysis. The sample preparation and microarray hybridization were performed according to the manufacturer's instructions with minor modifications. Briefly, mRNA was purified from total RNA following the removal of rRNA using an mRNA-ONLY ${ }^{\mathrm{TM}}$ eukaryotic mRNA isolation kit (Epicentre Biotechnologies, Madison, WI, USA). Subsequently, each sample was amplified and transcribed into fluorescent cRNA along the entire length of the transcripts without 3 ' bias using a random priming method. The labeled
Table I. Demographical characteristics of 90 cases of non-small cell lung cancer.

\begin{tabular}{lc}
\hline Parameter & Year/number \\
\hline Age (years) & $64.5(37-80)$ \\
Gender (female/male) & $50 / 40$ \\
Histological grade & \\
Well-differentiated carcinoma & 13 \\
Well- to moderately differentiated carcinoma & 15 \\
Moderately differentiated carcinoma & 32 \\
Moderately to poorly differentiated carcinoma & 12 \\
Poorly differentiated carcinoma & 18 \\
TNM clinical stage & \\
Ia & 22 \\
Ib & 36 \\
IIa & 11 \\
IIb & 5 \\
IIIa & 16 \\
\hline
\end{tabular}

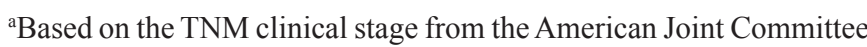
on Cancer (AJCC) and the Union for International Cancer Control (UICC) in 2002 (33).

cRNAs were hybridized onto a Human lncRNA Array v3.0 (8x60 K; Arraystar, Rockville, MD, USA), designed for 30,586 lncRNAs and 26,109 coding transcripts. The lncRNAs were carefully constructed using the most highly respected public transcriptome databases, including Refseq (http://www.ncbi. nlm.nih.gov/refseq/), UCSC Known Genes (http://www. biomedsearch.com/nih/UCSC-Known-Genes/16500937.html) and GENCODE (http://www.gencodegenes.org/) as well as landmark publications (20-22). Each transcript was accurately identified by a specific exon or splice junction probe. Positive probes for housekeeping genes and negative probes were also printed onto the array for hybridization quality control. After washing the slides, the arrays were scanned using the $\mathrm{G} 2505 \mathrm{C}$ scanner (Agilent Technologies, Inc.), and the acquired array images were analyzed with the Feature Extraction software (version 11.0.1.1, Agilent Technologies, Inc.). Quantile normalization and subsequent data processing were performed using the GeneSpring GX v12.0 software package (Agilent Technologies, Inc.). The microarray was performed by KangChen Bio-tech, Shanghai, China.

Functional group analysis. Gene ontology (GO) analysis was derived from Gene Ontology (www.geneontology.org), which provides three structured networks of defined terms that describe gene product attributes. The P-value denotes the significance of GO term enrichment in the differentially expressed mRNA list $(\mathrm{P} \leq 0.05$ was considered to indicate a statistically significant difference). Pathway analysis was also carried out for the differentially expressed mRNAs based on the latest Kyoto Encyclopedia of Genes and Genomes (KEGG; http://www.genome.jp/kegg/) database. This analysis allowed us to determine the biological pathway 
Table II. Upregulated and downregulated long-chain non-coding RNAs in non-small cell lung cancer.

\begin{tabular}{|c|c|c|c|}
\hline Probe name & $\begin{array}{c}\text { FC (abs) } \\
\text { (NSCLC vs normal lung tissue) }\end{array}$ & Regulation & Gene symbol \\
\hline ASHGA5P051906 & 2.3496380 & Up & RP11-412P11.1 \\
\hline ASHGA5P050658 & 3.3447275 & Up & AC140481.7 \\
\hline ASHGA5P045969 & 2.3421350 & Up & AK129672 \\
\hline ASHGA5P037374 & 3.0890374 & $\mathrm{Up}$ & AP001469.9 \\
\hline ASHGA5P027700 & 2.3656695 & $\mathrm{Up}$ & FLJ31485 \\
\hline ASHGA5P020784 & 5.6349115 & Up & RP11-909N17.3 \\
\hline ASHGA5P035023 & 2.8399296 & $\mathrm{Up}$ & XLOC_002399 \\
\hline ASHGA5P055971 & -3.3054008 & Down & XLOC_012542 \\
\hline ASHGA5P047263 & -3.5518806 & Down & RP11-445K13.2 \\
\hline ASHGA5P031003 & -4.0572240 & Down & CTA-363E6.2 \\
\hline ASHGA5P039685 & -2.9653406 & Down & HSP90AA6P \\
\hline ASHGA5P026985 & -2.3325500 & Down & RP11-264F23.3 \\
\hline ASHGA5P055824 & -4.2268586 & Down & GPC5-AS1 \\
\hline ASHGA5P040177 & -2.7432818 & Down & ВX004987.5 \\
\hline
\end{tabular}

NSCLC, non-small cell lung cancer.

for which a significant enrichment of differentially expressed mRNAs existed.

$R T-q P C R$. Total RNA was extracted from frozen NSCLC tissues with TRIzol reagent (Invitrogen Life Technologies) and then reverse transcribed using an RT reagent kit (Thermo Fisher Scientific, Waltham, MA, USA) according to the manufacturer's instructions. LncRNA expression in NSCLC tissues was measured by qPCR using SYBR Premix Ex Taq (Thermo Fisher Scientific) with an ABI 7000 instrument (Applied Biosystems, Inc., Foster City, NJ, USA). Two IncRNAs that were significantly expressed (RP11-385J1.2 and TUBA4B) were evaluated in all of the patients included in this study. Total RNA ( $2 \mathrm{mg}$ ) was transcribed to cDNA. PCR was performed in a total reaction volume of $20 \mu$, including $10 \mu \mathrm{l}$ SYBR Premix (2X), $2 \mu \mathrm{l}$ cDNA template, $1 \mu \mathrm{l}$ PCR forward primer $(10 \mathrm{mM}$; (5'-TGTCAGACTCTCGGGACCAT-3' for RP11-385J1.2 and 5'-AAAGTGCAACGTGCCATGTG-3' forTUBA4B), $1 \mu 1 \mathrm{PCR}$ reverse primer (10 mM; 5'-GATGCCACTGGAGTGTTGGA-3' for RP11-385J1.2 and 5'-CTCCACACTATCCATGCCCA-3' for TUBA4B) and $6 \mu \mathrm{l}$ double-distilled water. The qPCR reaction was performed with an initial denaturation step of $10 \mathrm{~min}$ at $95^{\circ} \mathrm{C}$, then $95^{\circ} \mathrm{C}(5 \mathrm{sec})$ and $60^{\circ} \mathrm{C}(30 \mathrm{sec})$ for a total of 40 cycles, with a final extension step at $72^{\circ} \mathrm{C}$ for $5 \mathrm{~min}$. All experiments were performed in triplicate and all samples were normalized to GAPDH. The median in each triplicate was used to calculate the relative lncRNA concentrations $(\Delta \mathrm{Ct}=\mathrm{Ct}$ median lncRNAs - Ct median GAPDH $)$. The fold changes in expression were calculated (23).

Statistical methods. The Shapiro-Wilk test was used to evaluate the distribution. Comparisons between two groups were tested using the Mann-Whitney U test for non-normal distribution. The fold change and Student's t-test were analyzed for statistical significance of the microarray results.
The false discovery rate was calculated to correct the P-value. The threshold value used to designate differentially expressed lncRNAs and mRNAs was a fold change of $\geq 2.0$ or $\leq 0.5$. $\mathrm{P}<0.05$ was considered to indicate a statistically significant difference. SPSS version 18.0 (SPSS Inc., Chicago, IL, USA) was used for statistical analysis.

\section{Results}

Overview of IncRNA expression profiles. To study the potential biological functions of lncRNAs in NSCLC, we examined the IncRNA and mRNA expression profiles in human NSCLC using microarray analysis (Fig. 1). For this analysis, authoritative data sources containing $>30,586$ lncR NAs were used. The expression profiles of 1,242 lncRNAs indicated that they were differentially expressed (fold change $\geq 2.0$ or $\leq 0.5$; $\mathrm{P}<0.05$ ) between NSCLC and normal lung samples. Among these, 541 lncRNAs were observed to be upregulated $>2$-fold in the NSCLC group compared with the normal lung group, while 701 lncRNAs were downregulated $>2$-fold $(\mathrm{P}<0.05$; Table II, Fig. 1A and B, Fig. 2A).

LncRNA classification and subgroup analysis. The expression profiles of 343 intergenic lncRNAs indicated that they were differentially expressed (fold change $\geq 2.0, \mathrm{P}<0.05$ ) between NSCLC and normal lung samples. Among these, 167 were upregulated and 176 were downregulated. Nearby coding genes that may be regulated by these lncRNAs were also identified (Table III). LncRNAs with enhancer-like function (lncRNA-a) were identified using GENCODE annotation. The expression profiles of 18 enhancer-like lncRNAs indicated that they were differentially expressed (fold change $\geq 2.0, \mathrm{P}<0.05$ ) between NSCLC and normal lung samples. Among these, seven were upregulated and 11 were downregulated. Nearby coding genes that may be regulated by these enhancer-like 
A

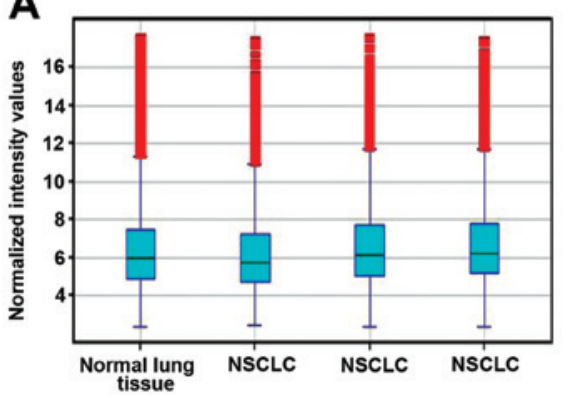

C

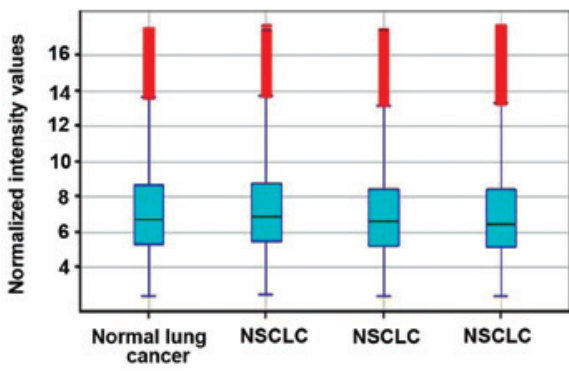

B

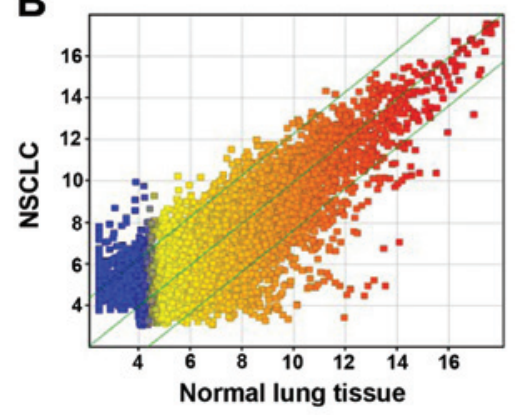

D

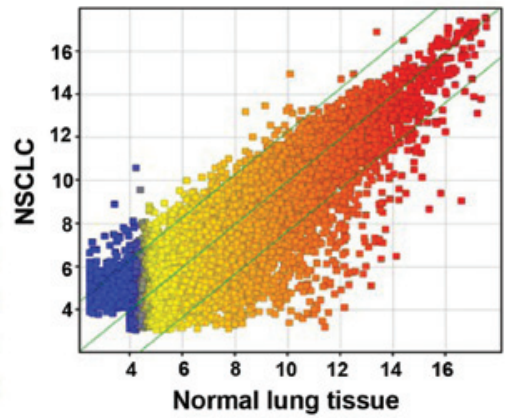

Figure 1. Distribution of a dataset in long-chain non-coding RNA (lncRNA) (A) and mRNA (B) profiles shown by box plot. Following normalization, the distributions of $\log 2$ ratios among the tested samples were almost the same. The scatter plot was used to assess the lncRNA (C) and mRNA (D) expression variation between non-small cell lung cancer (NSCLC) and normal lung arrays. The values of the X-and Y-axes in the scatter plot are averaged normalized values in each group ( $\log 2$-scaled). The lncRNAs above the top green line and below the bottom green line indicate $>$ three-fold change of lncRNAs between pairs.

A

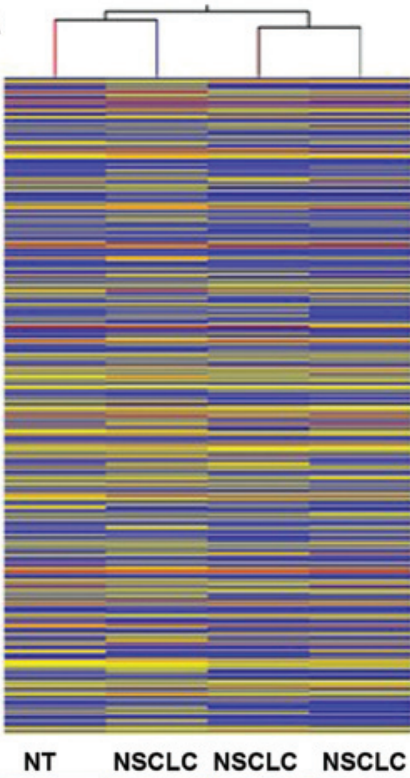

B

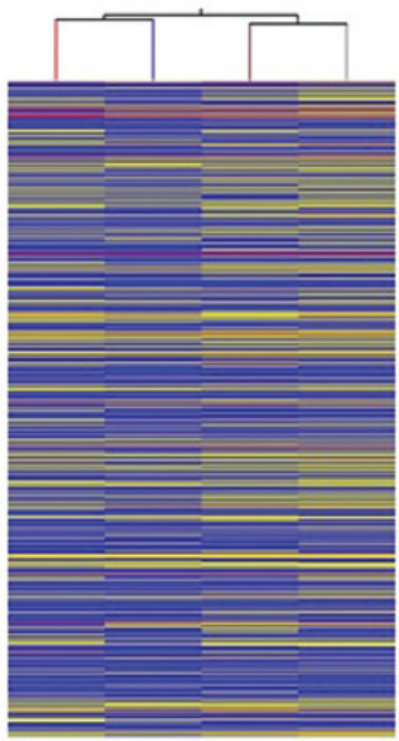

NT NSCLC NSCLC NSCLC

Figure 2. Heat map and hierarchical clustering of long-chain non-coding RNA (A) and mRNA (B) profile comparison between a non-small cell lung cancer (NSCLC) sample and a normal tissue (NT) sample.

lncRNAs were also identified (Table IV). Hox lncRNAs (lncRNAs transcribed from Hox loci lncRNAs) profiles: This table contains 83 HoxlncRNA clusters (data not shown).

Overview of $m R N A$ expression profiles. In total, 1,102 mRNAs were noted to be differentially expressed between NSCLC and normal lung samples, including 271 upregulated
mRNAs and 831 downregulated mRNAs (Fig. 1C and D, Fig. 2B).

$G O$ analysis. The genes corresponding to the downregulated mRNAs included 278 genes involved in biological processes, 75 genes involved in cellular components and 59 genes involved in molecular functions. The genes corresponding to 
Table III. Upregulated and downregulated long-chain non-coding RNAs (lncRNAs) in non-small cell lung cancer and nearby encoding genes regulated by lncRNAs.

\begin{tabular}{llclc}
\hline Seqname & Gene symbol & $\begin{array}{c}\text { Absolute fold } \\
\text { change lncRNAs }\end{array}$ & $\begin{array}{c}\text { Regulatory } \\
\text { lncRNAs }\end{array}$ \\
\hline ENST00000562902 & RP11-426C22.5 & -4.2223506 & Down & NM_007245 \\
ENST00000563624 & RP11-68I18.10 & 2.4656520 & Up & NM_003557 \\
ENST00000563624 & RP11-68I18.10 & 2.4656520 & Up & NM_207171 \\
ENST00000564524 & FAM157C & 2.4693956 & Up & ENST00000555147 \\
ENST00000564854 & RP13-514E23.1 & -3.2555604 & Down & NM_138980 \\
ENST00000565118 & ABCC6P1 & -2.4422970 & Down & NM_015161 \\
ENST00000565153 & RP11-297L17.2 & 2.5062720 & Up & NM_001080430 \\
ENST00000565862 & RP11-594N15.3 & -2.6900120 & Down & ENST00000263851 \\
ENST00000566420 & RP11-506E9.3 & -3.4461820 & Down & NM_153699 \\
ENST00000566942 & RP11-284N8.3 & -3.9437150 & Down & NM_001040033 \\
\hline
\end{tabular}

Table IV. Enhancer-like long-chain non-coding RNAs (lncRNAs) in non-small cell lung cancer and nearby encoding genes regulated by lncRNAs.

\begin{tabular}{|c|c|c|c|c|}
\hline Seqname & Gene symbol & $\begin{array}{c}\text { Absolute fold } \\
\text { change lncRNAs }\end{array}$ & $\begin{array}{l}\text { Regulatory } \\
\text { lncRNAs }\end{array}$ & Nearby gene \\
\hline ENST00000366140 & AC017076.5 & -2.3342237 & Down & NM_207315 \\
\hline ENST00000418076 & RP11-37E23.5 & -2.8438077 & Down & NM_001079691 \\
\hline ENST00000421619 & RP11-114B7.6 & 3.0627985 & $\mathrm{Up}$ & ENST00000373484 \\
\hline ENST00000421619 & RP11-114B7.6 & 3.0627985 & $\mathrm{Up}$ & NM_001145720 \\
\hline ENST00000428508 & RP11-353N4.1 & -2.7596264 & Down & NM_001123375 \\
\hline ENST00000433986 & RP11-261C10.3 & 2.8433535 & $\mathrm{Up}$ & NM_014812 \\
\hline ENST00000446476 & RP5-826L7.1 & -3.6936464 & Down & NM_152410 \\
\hline ENST00000446476 & RP5-826L7.1 & -3.6936464 & Down & NM_206853 \\
\hline ENST00000446476 & RP5-826L7.1 & -3.6936464 & Down & NM_206854 \\
\hline ENST00000453853 & RP11-342C24.8 & 2.8042965 & Up & ENST00000374325 \\
\hline
\end{tabular}

Table V. Pathway analysis of upregulated mRNA in non-small cell lung cancer.

\begin{tabular}{|c|c|c|c|c|}
\hline Pathway ID & Definition & Fisher P-value & $\begin{array}{l}\text { False } \\
\text { discovery rate }\end{array}$ & $\begin{array}{l}\text { Enrichment } \\
\text { score }\end{array}$ \\
\hline hsa05034 & Alcoholism - Homo sapiens (human) & $7.52732 \mathrm{E}-07$ & 0.000197968 & 6.123360 \\
\hline hsa05322 & $\begin{array}{l}\text { Systemic lupus erythematosus - } \\
\text { Homo sapiens (human) }\end{array}$ & $5.81856 \mathrm{E}-05$ & 0.007651410 & 4.235184 \\
\hline hsa05202 & $\begin{array}{l}\text { Transcriptional misregulation in cancer - } \\
\text { Homo sapiens (human) }\end{array}$ & 0.000123941 & 0.010865460 & 3.906786 \\
\hline hsa04110 & Cell cycle - Homo sapiens (human) & 0.003491957 & 0.229596200 & 2.456931 \\
\hline hsa04070 & $\begin{array}{l}\text { Phosphatidylinositol signaling system - } \\
\text { Homo sapiens (human) }\end{array}$ & 0.009181137 & 0.466312700 & 2.037104 \\
\hline hsa04744 & Phototransduction - Homo sapiens (human) & 0.010638310 & 0.466312700 & 1.973127 \\
\hline hsa05214 & Glioma - Homo sapiens (human) & 0.019614550 & 0.678238400 & 1.707422 \\
\hline hsa05212 & Pancreatic cancer - Homo sapiens (human) & 0.020630820 & 0.678238400 & 1.685483 \\
\hline hsa04114 & Oocyte meiosis - Homo sapiens (human) & 0.032740740 & 0.956757000 & 1.484912 \\
\hline hsa05203 & Viral carcinogenesis - Homo sapiens (human) & 0.046635330 & 1 & 1.331285 \\
\hline hsa04512 & $\begin{array}{l}\text { ECM-receptor interaction - } \\
\text { Homo sapiens (human) }\end{array}$ & 0.047875420 & 1 & 1.319887 \\
\hline
\end{tabular}




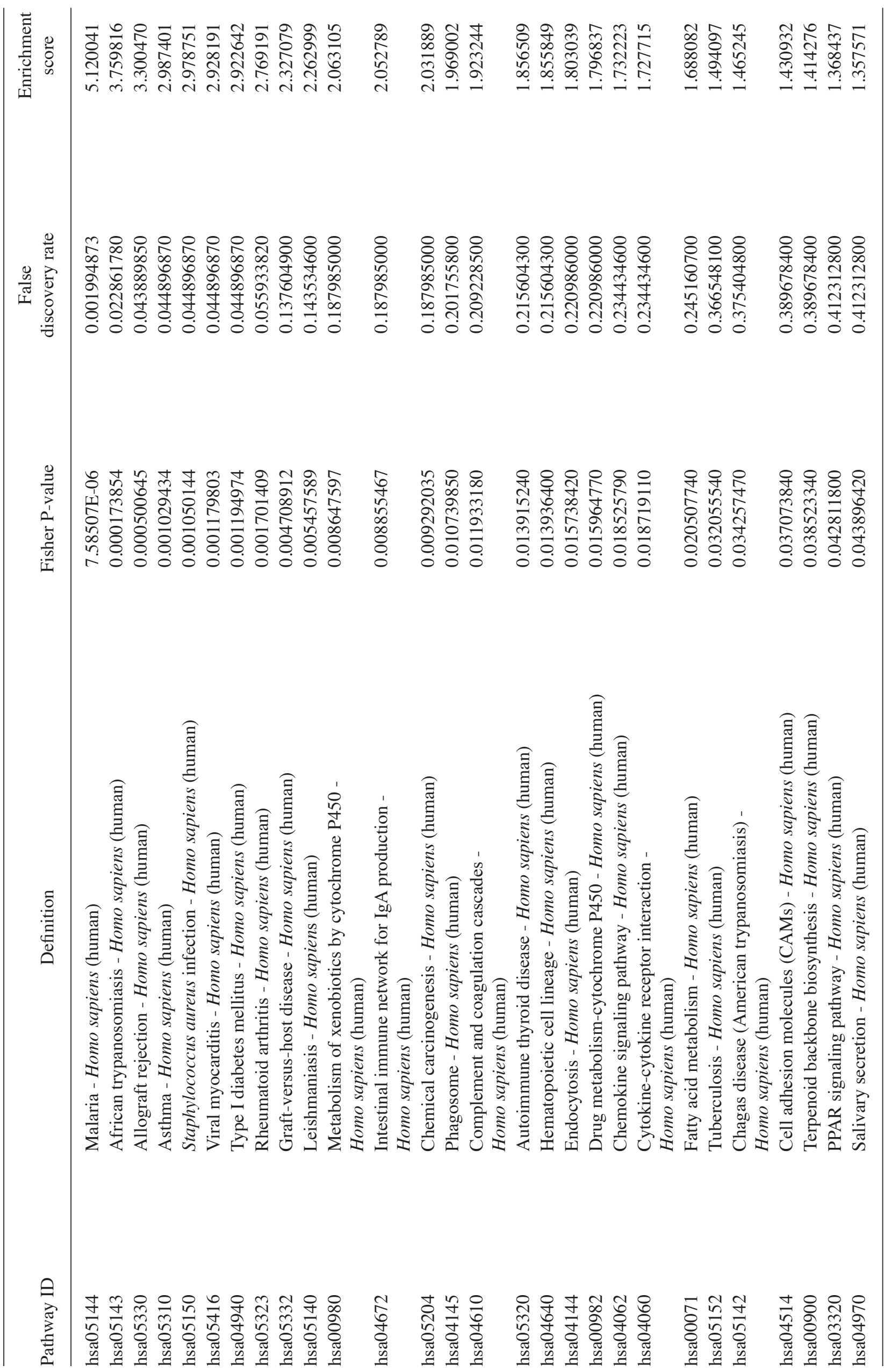




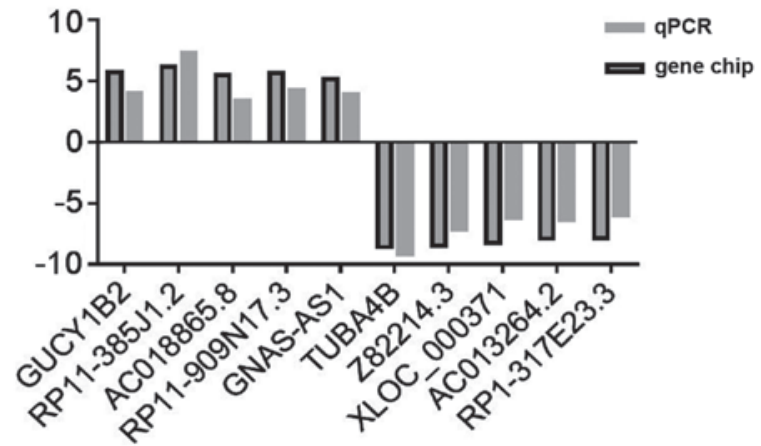

Figure 3. Comparison between gene chip data and reverse transcription-quantitative polymerase chain reaction (RT-qPCR) results. GUCY1B2, RP11-385J1.2, AC018865.8, RP11-909N17.3, GNAS-AS1, TUBA4B, Z82214.3, XLOC_000371, AC013264.2 and RP1-317E23.3 were determined to be differentially expressed in non-small cell lung cancer samples compared with normal tissue samples in six patients by microarray, and were validated by RT-qPCR. The heights of the columns in the chart represent the log-transformed median fold changes $(\mathrm{T} / \mathrm{N})$ in expression across the six patients for each of the four lncRNAs validated; the bars represent standard error. The validation results of the 19 lncRNAs indicate that the microarray data correlate well with the qPCR results.

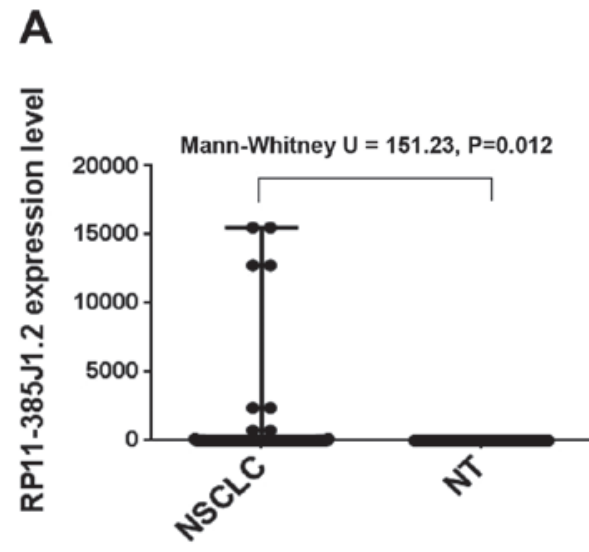

B

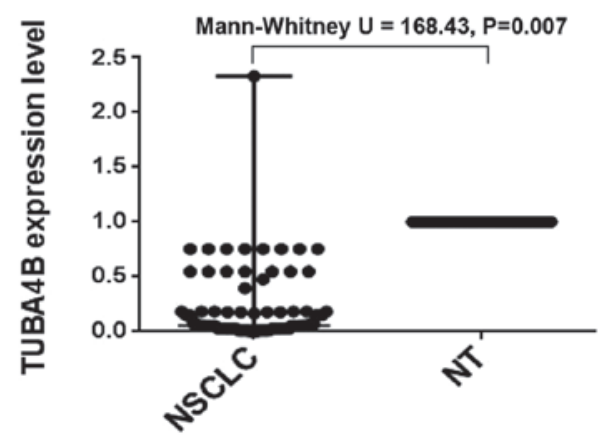

Figure 4. Expression levels of RP11-385J1.2 and TUBA4B between 105 non-small cell lung cancer (NSCLC) samples and normal tissue (NT) samples. (A) RP11-385J1.2 expression in NSCLC was significantly higher than in NT (Mann-Whitney U test=151.23, $\mathrm{P}=0.012$ ). (B) TUBA4B expression in NSCLC was significantly lower than in NT (Mann-Whitney U test=168.43, $\mathrm{P}=0.007)$.

the upregulated mRNAs included 246 genes involved in biological processes, 58 genes involved in cellular components and 66 genes involved in molecular functions.
Pathway analysis. Eleven upregulated pathways were identified, including ethanol metabolism, systemic lupus erythematosus, transcriptional misregulation in cancer and cell cycle pathways (Table V). Twenty-eight downregulated pathways were identified, including malaria, African trypanosomiasis and allograft rejection (Table VI).

$R T-q P C R$ validation. According to factors including the fold difference, gene locus and nearby encoding gene, we initially identified a number of significant candidate lncRNAs (including GUCY1B2, RP11-385J1.2, AC018865.8, RP11-909N17.3, GNAS-AS1, TUBA4B, Z82214.3, XLOC_000371, AC013264.2 and RP1-317E23.3) and verified the expression of these IncRNAs by RT-qPCR with GAPDH as the reference gene, by calculating the $2^{-\triangle \Delta C T}$ values. We observed that multiple lncRNAs in the microarray were consistent with the results of the RT-qPCR; see Fig. 3. RP11-385J1.2 and TUBA4B were the most markedly changed of these candidate lncRNAs from 90 NSCLC and normal lung tissue samples. As shown in Fig. 4, RP11-385J1.2 expression in NSCLC was significantly higher than in the adjacent tissues (Mann-Whitney $\mathrm{U}$ test $=151.23$, $\mathrm{P}=0.012$ ), while TUBA4B expression in NSCLC was significantly lower than in the adjacent tissues (Mann-Whitney $\mathrm{U}$ test=168.43, $\mathrm{P}=0.007$ ).

\section{Discussion}

According to the 2012 China Oncology Annual Report, the 2009 incidence and mortality of lung cancer was the highest among all cancers in male patients and the second highest among all cancers in female patients in China. LncRNAs play a significant role in a number of biological processes, including X-chromosome inactivation, gene imprinting and stem cell maintenance $(24,25)$. It has been confirmed that lncRNAs are one of the most significant factors controlling gene expression in cancer (26). LncRNAs including HOTAIR have been shown to play a crucial role in the development and progression of tumors (9). It has also been demonstrated that lncRNAs are differentially expressed in normal and tumor cells $(27,28)$. As lncRNAs constitute an essential class of gene expression regulatory factors, their aberrant expression would inevitably lead to abnormal gene expression levels, which may result in tumorigenesis.

In this study, we analyzed the lncRNA expression profile in the tissue of NSCLC patients to elucidate the potential role of lncRNAs in the pathogenesis of this disease. High-throughput microarray techniques revealed a set of differentially expressed lncRNAs, with 541 of those upregulated and 701 downregulated in NSCLC tissue compared with normal lung tissue. LncRNAs are usually divided into five categories: Sense, antisense, bidirectional, intronic and intergenic (29). LncRNAs are known to function via a variety of mechanisms; however, a common and significant function of lncRNAs is to alter the expression of nearby encoding genes by affecting the process of transcription (30) or directly playing an enhancer-like role $(31,32)$. In the present study, we increased the accuracy of target prediction by comparing differentially expressed mRNAs with differentially expressed lncRNAs. The lncRNA expression profiles indicated that 343 lncRNAs were differentially expressed (167 upregulated and 176 downregulated) between NSCLC and normal lung samples. The expression profiles included 18 differentially expressed enhancer-like lncRNAs, with seven upregulated and 
11 downregulated. Nearby coding genes that may be regulated by lncRNAs and enhancer-like lncRNAs were also identified. In addition, we performed HOX cluster profiling of lncRNAs and coding transcripts.

In order to obtain insights into lncRNA target gene function, GO analysis and KEGG pathway annotation were applied to the lncRNA target gene pool. GO analysis revealed that the number of genes corresponding to downregulated mRNAs was larger than that corresponding to upregulated mRNAs. KEGG annotation revealed 11 upregulated pathways (including ethanol metabolism, systemic lupus erythematosus, transcriptional misregulation in cancer and cell cycle pathways) and 28 downregulated pathways (including malaria, African trypanosomiasis and allograft rejection). These pathways may play significant roles in the occurrence and development of NSCLC. Ten IncRNAs identified in the microarray analysis were confirmed by RT-qPCR to be aberrantly expressed in NSCLC tissues. Among these lncRNAs, RP11-385J1.2 was the most markedly upregulated and TUBA4B was the most markedly downregulated. This result suggests that RP11-385J1.2 and TUBA4B may contribute to the development of NSCLC; further study of the biological function of RP11-385J1.2 and TUBA4B will be required to confirm this.

In conclusion, the present study revealed a set of lncRNAs with differential expression in NSCLC compared with normal lung tissue. Furthermore, it was demonstrated that RP11-385J1.2 and TUBA4B may contribute to the development of NSCLC. Further investigation of the lncRNAs identified in this study will likely provide insights into their biological functions and their association with NSCLC.

\section{Acknowledgements}

This study was financially supported by the National Natural Science Foundation of China (81401736 and 81271906) and Wenzhou Municipal Science and Technology Bureau, China (Y20110041 and Y20130170). The authors thank all donors who donated to the microarray service at KangChen Bio-tech, Shanghai, China.

\section{References}

1. Jemal A, Murray T, Ward E, Samuels A, Tiwari RC, Ghafoor A, Feuer EJ and Thun MJ: Cancer statistics, 2005. CA Cancer J Clin 55: 10-30, 2005.

2. Gridelli C, Rossi A and Maione P: Treatment of non-small-cell lung cancer: state of the art and development of new biologic agents. Oncogene 22: 6629-6638, 2003.

3. Stewart DJ: Tumor and host factors that may limit efficacy of chemotherapy in non-small cell and small cell lung cancer. Crit Rev Oncol Hematol 75: 173-234, 2010.

4. Chen CH,Lai JM, Chou TY, Chen CY, Su LJ,Lee YC, et al: VEGFA upregulates FLJ10540 and modulates migration and invasion of lung cancer via PI3K/AKT pathway. PloS One 4: e5052, 2009.

5. Ogawa E, Takenaka K, Katakura H, Adachi M, Otake Y, Toda Y, et al: Perimembrane Aurora-A expression is a significant prognostic factor in correlation with proliferative activity in non-small-cell lung cancer (NSCLC). Ann Surg Oncol 15: 547-554, 2008.

6. Rachet B, Woods LM, Mitry E, Riga M, Cooper N, Quinn MJ, Coleman MP, et al: Cancer survival in England and Wales at the end of the 20th century. Br J Cancer 99: S2-S10, 2008.

7. Ponting CP, Oliver PL and Reik W: Evolution and functions of long noncoding RNAs. Cell 136: 629-641, 2009.

8. Dinger ME, Pang KC, Mercer TR, Crowe ML, Grimmond SM and Mattick JS: NRED: a database of long noncoding RNA expression. Nucleic Acids Res 37: D122-D126, 2009.
9. Gupta RA, Shah N, Wang KC, Kim J, Horlings HM, Wong DJ, Chang HY, et al: Long non-coding RNA HOTAIR reprograms chromatin state to promote cancer metastasis. Nature 464: 1071-1076, 2010.

10. Wapinski $\mathrm{O}$ and Chang HY: Long noncoding RNAs and human disease Trends Cell Biol 21: 354-361, 2011.

11. Fu X, Ravindranath L, Tran N, Petrovics G and Srivastava S: Regulation of apoptosis by a prostate-specific and prostate cancer-associated noncoding gene, PCGEM1. DNA Cell Biol 25: 135-141, 2006.

12. Zhang H, Chen Z, Wang X, Huang Z, He Z and Chen Y: Long non-coding RNA: a new player in cancer. J Hematol Oncol 6: $37,2013$.

13. Hauptman $\mathrm{N}$ and Glavac D: Long non-coding RNA in cancer. Int J Mol Sci 14: 4655-4669, 2013.

14. Chen G, Wang Z, Wang D, Qiu C, Liu M, Chen X, Cui Q, et al: LncRNADisease: a database for long-non-coding RNA-associated diseases. Nucleic Acids Res 41: D983-D986, 2013.

15. Gibb EA, Brown CJ and Lam WL: The functional role of long non-coding RNA in human carcinomas. Mol Cancer 10: 38, 2011.

16. Ji P, Diederichs S, Wang W, Boing S, Metzger R, Schneider PM, Muller-Tidow C, et al: MALAT-1, a novel noncoding RNA, and thymosin beta4 predict metastasis and survival in early-stage non-small cell lung cancer. Oncogene 22: 8031-8041, 2003.

17. Thai P, Statt S, Chen $\mathrm{CH}$, Liang E, Campbell $\mathrm{C}$ and $\mathrm{Wu} \mathrm{R}$ : Characterization of a novel long noncoding RNA, SCAL1, induced by cigarette smoke and elevated in lung cancer cell lines. Am J Respir Cell Mol Biol 49: 204-211, 2013.

18. Yang Y, Li H, Hou S, Hu B, Liu J and Wang J: The noncoding RNA expression profile and the effect of lncRNA AK126698 on cisplatin resistance in non-small-cell lung cancer cell. PloS One 8: e65309, 2013.

19. Han L, Kong R, Yin DD, Zhang EB, Xu TP, De W and Shu YQ: Low expression of long noncoding RNA GAS6-AS1 predicts a poor prognosis in patients with NSCLC. Med Oncol 30: 694, 2013.

20. Fritah S, Niclou SP and Azuaje F: Databases for lncRNAs: a comparative evaluation of emerging tools. RNA 20: 1655-1665, 2014.

21. Chakraborty S, Deb A, Maji RK, Saha S and Ghosh Z: LncRBase: an enriched resource for lncRNA information. PLoS One 9: e108010, 2014.

22. Quek XC, Thomson DW, Maag JL, et al: lncRNAdb v2.0: expanding the reference database for functional long noncoding RNAs. Nucleic Acids Res: Oct 20, 2014.

23. Ren S, Peng Z, Mao JH, Yu Y, Yin C, Gao X, Sun Y, et al: RNA-seq analysis of prostate cancer in the Chinese population identifies recurrent gene fusions, cancer-associated long noncoding RNAs and aberrant alternative splicings. Cell Res 22: 806-821, 2012.

24. Mercer TR, Dinger ME and Mattick JS: Long non-coding RNAs: insights into functions. Nature reviews. Genetics 10: 155-159, 2009.

25. Wang KC and Chang HY: Molecular mechanisms of long noncoding RNAs. Mol Cell 43: 904-914, 2011.

26. Khachane AN and Harrison PM: Mining mammalian transcript data for functional long non-coding RNAs. PloS One 5: e10316, 2010.

27. Lai MC, Yang Z,Zhou L, et al: Long non-coding RNA MALAT-1 overexpression predicts tumor recurrence of hepatocellular carcinoma after liver transplantation. Med Oncol 29: 1810-1816, 2012.

28. Braconi C, Kogure T, Valeri N, et al: microRNA-29 can regulate expression of the long non-coding RNA gene MEG3 in hepatocellular cancer. Oncogene 30: 4750-4756, 2011.

29. $\mathrm{Li} \mathrm{CH}$ and Chen Y: Targeting long non-coding RNAs in cancers: progress and prospects. Int J Biochem Cell Biol 45: 1895-1910, 2013.

30. Mattick JS and Gagen MJ: The evolution of controlled multitasked gene networks: the role of introns and other noncoding RNAs in the development of complex organisms. Mol Biol Evol 18: 1611-1630, 2001.

31. Mattick JS: Linc-ing Long noncoding RNAs and enhancer function. Dev Cell 19: 485-486, 2010.

32. Orom UA, Derrien T, Beringer M, Gumireddy K, Gardini A, Bussotti G, Shiekhattar R, et al: Long noncoding RNAs with enhancer-like function in human cells. Cell 143: 46-58, 2010.

33. Edge SB and Compton CC: The American Joint Committee on Cancer: the 7th edition of the AJCC cancer staging manual and the future of TNM. Ann Surg Oncol 17: 1471-1474, 2010. 